This is the author's final, peer-reviewed manuscript as accepted for publication. The publisher-formatted version may be available through the publisher's web site or your institution's library.

\title{
The enterohemorrhagic Escherichia coli effector protein NleF binds mammalian Tmp21
}

Rachel L. Olsen, Frank Echtenkamp, Dilyara Cheranova, Wanyin Deng, B. Brett Finlay, and Philip R. Hardwidge

\section{How to cite this manuscript}

If you make reference to this version of the manuscript, use the following information:

Olsen, R. L., Echtenkamp, F., Cheranova, D., Deng, W., Finlay, B. B., \& Hardwidge, P. R. (2013). The enterohemorrhagic Escherichia coli effector protein NleF binds mammalian Tmp21. Retrieved from http://krex.ksu.edu

\section{Published Version Information}

Citation: Olsen, R. L., Echtenkamp, F., Cheranova, D., Deng, W., Finlay, B. B., \& Hardwidge, P. R. (2013). The enterohemorrhagic Escherichia coli effector protein NleF binds mammalian Tmp21. Veterinary Microbiology, 164(1-2), 164-170.

Copyright: (C) 2013 Elsevier B.V.

Digital Object Identifier (DOI): doi:10.1016/j.vetmic.2013.01.028

Publisher's Link: http://www.sciencedirect.com/science/article/pii/S0378113513000801

This item was retrieved from the K-State Research Exchange (K-REx), the institutional repository of Kansas State University. K-REx is available at http://krex.ksu.edu 
Rachel L. Olsen ${ }^{1}$, Frank Echtenkamp ${ }^{2}$, Dilyara Cheranova ${ }^{1,3}$, Wanyin Deng ${ }^{4}$, B. Brett Finlay ${ }^{4}$, and Philip R. Hardwidge 5* $^{*}$

6 1) Department of Microbiology, Molecular Genetics, and Immunology, University of Kansas Medical 7 Center, Kansas City, KS, USA.

8 2) Department of Cell and Developmental Biology, University of Illinois, Urbana-Champaign, Urbana, 9 Illinois, USA.

10 3) Department of Pediatrics, Children's Mercy Hospital, Kansas City, MO, USA.

11 4) Michael Smith Laboratories, University of British Columbia, Vancouver, BC, Canada.

12 5) Department of Diagnostic Medicine/Pathobiology, Kansas State University, Manhattan, KS, USA.

*To whom correspondence should be addressed:

15 Philip R. Hardwidge

340 Coles Hall, Kansas State University, Manhattan, KS 66506

17 Email: hardwidg@gmail.com

18 Phone: 785-532-2506

19 Fax: 785-532-4851

21 Running title: NleF binds Tmp21

Keywords: effector, enterohemorrhagic E. coli, NleF, Tmp21, type III secretion

Abbreviations: A/E, attaching and effacing; BiFC, bimolecular fluorescence complementation; EHEC, enterohemorrhagic E. coli; EPEC, enteropathogenic E. coli; ER, endoplasmic reticulum; LEE, locus of enterocyte effacement; NleF, non-LEE encoded protein F; STEC, Shiga-like toxin-producing E. coli; T3SS, type III secretion system 
29 Abstract. The human pathogens enterohemorrhagic and enteropathogenic Escherichia coli (EHEC and

30 EPEC), as well as the mouse pathogen Citrobacter rodentium encode type III secretion system (T3SS)

31 effector proteins to promote their survival in the infected host. The mechanisms of action and the host

32 targets of T3SS effectors are under active investigation because of their importance to bacterial virulence.

33 The non-locus of enterocyte effacement (LEE)-encoded protein F, NleF, contributes to $E$. coli and C.

34 rodentium colonization of piglets and mice, respectively. Here we sought to characterize the host binding

35 partners of NleF. Using a yeast two-hybrid screen, we identified Tmp21, a type-I integral membrane

36 protein and COPI-vesicle receptor involved in trans-Golgi network function, as an NleF-binding partner.

37 We confirmed this interaction using immunoprecipitation and bimolecular fluorescence complementation

38 (BiFC). We expressed a temperature-sensitive vesicular stomatitis virus glycoprotein (tsVSVG) to monitor

39 protein trafficking and determined that NleF slows the intracellular trafficking of tsVSVG from the 40 endoplasmic reticulum to the Golgi. 
42 Introduction. Enterohemorrhagic Escherichia coli (EHEC) and other Shiga-like toxin-producing E. coli

43 (STEC) are endemic health threats and major causes of food borne disease (Clarke, 2001). These human

44 pathogens cause hemorrhagic colitis and are a leading cause of pediatric renal failure. In addition to the

45 Shiga-like toxin, STEC encode a large number of virulence proteins, which they translocate into intestinal

46 epithelial cells using a type III secretion system [T3SS, (Cornelis, 2010)], a mechanism conserved among

47 the other attaching/effacing (A/E) pathogens, enteropathogenic E. coli (EPEC) and Citrobacter rodentium.

48 While it is known that these translocated proteins (effectors) subvert host cell function to promote diarrheal

49 disease and bacterial transmission, the biochemical mechanisms and host targets of many of these

50 effectors are unknown.

The mammalian protein secretory pathway relies on vesicular trafficking to transport cargo

between different organelles. Transport vesicles composed of protein coat complexes known as COPI and

COPII mediate protein trafficking between the endoplasmic reticulum (ER) and Golgi (Wessels et al., 2006). Proteins are transported from the ER to the Golgi (anterograde transport) by COPII carrier vesicles that bud from the ER (Kaiser and Ferro-Novick, 1998). Proteins arriving at the Golgi are modified and sorted into transport vesicles destined for the plasma membrane (PM), the endo/lysosomal system, or to secretory granules. A subset of proteins is recycled to the ER from the Golgi (retrograde transport) in COPI vesicles (Orci et al., 2000).

Bacterial exploitation of eukaryotic secretory pathways is of interest. For example, Salmonella recruits exocytic transport vesicles to the Salmonella-containing vacuole, possibly to interfere with antigen presentation (Kuhle et al., 2006). Brucella abortus utilizes the endoplasmic reticulum (ER) GTPase Sar1 62 for intracellular replication (Celli et al., 2005). The E.coli effector NleA inhibits COPII-dependent protein 63 export from the ER by binding to Sec24 (Kim et al., 2007).

We previously identified the NleF protein in a proteomic screen of EHEC and C. rodentium secreted proteins (Deng et al., 2004). NleF is found in the E. coli lineages associated with human disease 66 (Zhang et al., 2007) and with epidemic potential (Coombes et al., 2008). NleF is present with $100 \%$ amino acid identity in EPEC E2348/69 and an ortholog is also found in C. rodentium. Our previous experiments demonstrated that NleF is a T3SS-translocated effector (Echtenkamp et al., 2008). Infection experiments 
70 colonization of the host (Echtenkamp et al., 2008). The goal of this study was to determine the mammalian

71 binding partner of NleF. We demonstrate here that NleF binds to the human Tmp21 protein and 72 subsequently disrupts intracellular protein trafficking.

73 
Materials and Methods

75 Chemicals and antibodies. Chemicals and antibodies were used according to manufacturer's recommendations. GFP and Tmp21 antibodies were obtained from Cell Signaling. Golgin-97 antibody was obtained from Invitrogen. Calnexin, HA, and His antibodies were obtained from Sigma. NleF antiserum was described previously (Echtenkamp et al., 2008). pEGFP-VSVG was provided by J. LippincottSchwartz [Addgene \#11912; (Presley et al., 1997)].

Bacterial strains, cell culture, and infection experiments. The bacterial strains and plasmids used in this study are described in Table 1. HeLa cells were maintained in Dulbecco's modified Eagle's medium (DMEM) supplemented with $10 \%$ fetal bovine serum. Cells were transfected using TransPass (New England Biolabs). Bacteria were cultured in Luria-Bertani broth at $37^{\circ} \mathrm{C}$ for $18 \mathrm{~h}$ without shaking. Overnight LB cultures were diluted 1:10 into DMEM, followed by a further incubation for $3 \mathrm{~h}$ at $37^{\circ} \mathrm{C}, 5 \%$ $\mathrm{CO}_{2}$. Cell culture medium was replaced with DMEM prior to infection and bacteria were added at a multiplicity of infection of 25-50.

Protein purification. NleF and Tmp21 were cloned into pFLAG-CTC and pET28a, respectively, and expressed in E. coli BL21(DE3). Bacterial cultures were grown to an $\mathrm{OD}_{600}$ of 0.3 and then induced with 1 mM IPTG for 2 h. Cells were centrifuged, lysed by sonication, applied to $\alpha$-FLAG M2 beads and Ni-NTA agarose, respectively, and incubated at $4{ }^{\circ} \mathrm{C}$ overnight. After washing, proteins were eluted with either 0.1 M glycine $\mathrm{HCl}, \mathrm{pH} 3.5$, or with imidazole and then analyzed on SDS-12 \% PAGE.

Yeast two-hybrid assay. NleF was cloned into the yeast two-hybrid GAL4 DNA binding domain vector pGBKT7 to generate the 'bait' plasmid. The NleF bait was used to screen a pre-transformed human HeLa cell cDNA library for proteins interacting with NleF according to protocols in the BD Matchmaker Pretransformed Libraries User Manual (Clontech). Yeast clones containing library plasmids encoding human proteins interacting with NleF were purified by restreaking on selective media and retested for growth phenotypes. $\beta$-galactosidase activity was calculated using equation 1 , where $t$ refers to the incubation time (min) and $v$ refers to the concentration factor. Eq. 1: $\beta-g a l=\left(1000 * O D_{450}\right) /\left(t^{*} v * O D_{600}\right)$

Pull down assay. Purified proteins $(-20 \mu \mathrm{g})$ were applied to $\alpha$-FLAG M2 beads and incubated with rotation for $5 \mathrm{~h}$ at $4{ }^{\circ} \mathrm{C}$. Beads were washed three times with PBS and resuspended in SDS-PAGE buffer.

101 The samples were interrogated for the presence of His- and FLAG-tagged proteins by immunoblotting. 
102 Immunoblotting. Cells were lysed in RIPA buffer [150 mM NaCl, $50 \mathrm{mM}$ Tris pH $8.0,0.5 \%$ sodium

103 deoxycholate, $0.1 \%$ SDS, $1 \%$ Nonident P-40], incubated on ice for 30', and centrifuged. Proteins were

104 resolved by SDS-PAGE, transferred to nitrocellulose membranes, blocked in Odyssey blocking buffer (Li-

105 Cor) and then probed with appropriate primary and secondary antibodies. After rinsing in PBS, blots were

106 imaged using an Odyssey infrared imaging system.

107 Bimolecular fluorescence complementation. HeLa cells were co-transfected with two BiFC plasmids 108 (250 ng each) representing NleF and Tmp21 sequences cloned as fusions to the N- or C-terminus of 109 Venus eYFP (designated VN and VC). The fluorescence derived from BiFC was visualized using Eclipse

110 80i fluorescence microscope (Nikon) after $24 \mathrm{~h}$ incubation and was quantified using a fluorescence plate

111 reader with appropriate filters (excitation: 500/20 nm; emission: 535/30 nm).

112 VSVG trafficking and immunofluorescence microscopy. HeLa cells were grown on glass coverslips in

113 24-well tissue culture plates. For VSVG experiments, cells were transfected in 4 replicates with pEGFP-

114 VSVG in the presence or absence of NleF-HA. After $24 \mathrm{~h}$, one replicate was left at $37^{\circ} \mathrm{C}$ while the other 115 replicates were transferred to $19{ }^{\circ} \mathrm{C}, 32^{\circ} \mathrm{C}$, or $40^{\circ} \mathrm{C}$. Three hours after temperature shift, cells were rinsed 1163 times with PBS, fixed in $3.7 \%$ formaldehyde and permeabilized in $0.2 \%$ saponin in PBS, blocked with $11710 \%$ goat serum, and incubated with primary antibodies for $1 \mathrm{~h}$ at room temperature. Cells were washed 118 with PBS and probed with Alexa Fluor-conjugated secondary antibodies for $1 \mathrm{~h}$. Coverslips were mounted 119 in Mowiol and samples were visualized using Eclipse 80i fluorescence or Eclipse C1Si confocal 120 microscopes (Nikon).

121 Statistical analyses. $\beta$-galactosidase data were analyzed using t-tests. BiFC data were analyzed using 122 one-way ANOVA. VSVG trafficking data were evaluated with Fisher's exact test. p-values $<0.05$ were 123 considered significant. 
127 NleF binds to Tmp21. We used a yeast two-hybrid ( $\mathrm{Y} 2 \mathrm{H})$ screening assay to determine the mammalian

128 binding partners of NleF. EHEC nleF was cloned as a fusion to the Gal4 DNA-binding domain (DBD) and

129 co-expressed in the yeast reporter strain AH109 with a HeLa cell cDNA library. Co-transformants were

130 plated on synthetic quadruple-dropout (QDO) medium to select for interactions between NleF and a library

131 component that activated the transcriptional reporter. We isolated 153 colonies after $21 \mathrm{~d}$ growth on QDO

132 plates, 26 of which had a high level of reporter gene activation as measured in $\beta$-galactosidase assays

133 (data not shown).

134 We identified 3 different human cDNA sequences (Tmp21, CD151, PAIP2) that may encode

135 proteins that interact with NleF. Among these, we further studied Tmp21 (also named p23/p24d) as a 136 putative NleF binding partner. We confirmed the Tmp21-NleF interaction using direct $\mathrm{Y} 2 \mathrm{H}$ assays and by 137 quantifying $\beta$-galactosidase activity resulting from NleF-Tmp21 co-expression (Fig. 1A).

138 Tmp21 is a 219 amino acid integral type I transmembrane protein that functions as an integral 139 receptor for the COPI-vesicle coat (Blum et al., 1999). Tmp21 is a member of the p24 140 (p24/gp25L/emp24/Erp) protein family. These proteins provide cargo receptors to proteins (Anantharaman 141 and Aravind, 2002) and regulate protein packaging into COPI vesicles in concert with a small GTPase, the 142 ADP-ribosylation factor 1 (Arf1). p24 proteins are assembled into heteromeric complexes that cycle 143 between the ER and the Golgi and recruit Arf1 in early stages of vesicle formation (Gommel et al., 2001). 144 p24 proteins thus play active roles in retrograde protein transport from Golgi to ER by facilitating the 145 formation of COPI-coated vesicles (Aguilera-Romero et al., 2008).

146 By expressing and purifying recombinant forms of NleF and Tmp21 in E. coli BL21(DE3) and then 147 using these proteins in pulldown assays, we confirmed that NleF and Tmp21 bind directly in vitro (Fig. 148 1B). We also used immunofluorescence microscopy to determine the extent of NleF-Tmp21 colocalization. 149 To do this, we used polyclonal NleF antisera (Echtenkamp et al., 2008) to detect NleF after its 150 translocation into HeLa cells during C. rodentium infection (Fig. 1C). Co-staining for Tmp21 revealed that 151 both proteins colocalized in a perinuclear location (Fig. 1D).

152 BiFC. To determine whether NleF and Tmp21 interact when they are co-expressed in mammalian cells, 153 we used bimolecular fluorescence complementation (BiFC) assays. This technology utilizes the 
reconstitution of two fragments of the enhanced yellow fluorescent protein (eYFP) to generate a

155 fluorescent signal at the site of the protein-protein interaction under investigation (Hu et al., 2002). We

156 generated protein chimeras with split $\mathrm{N}$ - and C-terminal fragments (VN and VC, respectively) of eYFP

157 fused to either NleF or Tmp21 (Fig. 2A). Co-expressing eYFP chimeras of Tmp21 and NleF reconstituted

158 YFP fluorescence, to a similar magnitude as the reconstitution of the actin positive control (Fig. 2B). By

159 contrast, transfecting individual plasmids did not reconstitute YFP fluorescence (Fig. 2B), suggesting that

160 NleF binds to Tmp21 in mammalian cells.

161

The N-terminal luminal domain of Tmp21 mediates cargo uptake into transport vesicles, whereas

162 the KKLIE cytoplasmic tail at the Tmp21 carboxy-terminus mediates COPI-dependent transport vesicle

163 formation (Blum and Lepier, 2008). To map the binding domain of NleF on Tmp21, we carried out a BiFC

164 study with C-terminal deletions of NleF. These experiments revealed that deleting the NleF C-terminus

165 beyond amino acid 84 eliminated NleF binding to full length Tmp21, as indicated by loss of fluorescence

166 with the sequentially truncated constructs (Fig. 2C). A similar BiFC analysis revealed that the C-terminal

167 region of Tmp21, amino acids 63-180, was required for binding to NleF (Fig. 2D). Overall, these data

168 suggest that NleF and Tmp21 associate through their respective C-termini.

169 NleF alters VSVG trafficking. We tested the hypothesis that NleF binding to Tmp21 would cause defects

170 in protein trafficking by characterizing the localization of a vesicular stomatitis virus glycoprotein (VSVG)-

171 GFP fusion as a function of NleF expression and of temperature. VSVG localization is commonly used to

172 study mammalian protein trafficking (Wessels et al., 2005). This glycoprotein is essential for viral envelope

173 fusion with the host PM and traffics intracellularly via the ER and Golgi (Lippincott-Schwartz et al., 2000).

174 VSVG-GFP transport can be manipulated by incubating cell cultures at different temperatures

175 (Toomre et al., 1999). If incubated at $40{ }^{\circ} \mathrm{C}$, VSVG-GFP becomes reversibly misfolded and retained in the

176 ER. If shifted to $19{ }^{\circ} \mathrm{C}$, VSVG-GFP refolds and can be transported to the TGN. A further temperature shift

177 to $32^{\circ} \mathrm{C}$ allows subsequent trafficking to the PM (Fig. 3A).

We first transfected HeLa cells with VSVG-GFP and then evaluated VSVG-GFP localization using

179 immunofluorescence microscopy. As expected, when cells were shifted from $37^{\circ} \mathrm{C}$ to $40{ }^{\circ} \mathrm{C}$, VSVG-GFP

180 fluorescence became localized primarily in the ER, as shown by its colocalization with the ER protein

181 calnexin (Fig. 3B). When cells were subsequently shifted to $19{ }^{\circ} \mathrm{C}$, VSVG-GFP localization with calnexin 
was reduced and VSVG-GFP was primarily redistributed to the Golgi, as shown by its colocalization with

183 the Golgi protein golgin-97 (Fig. 3C). Incubating cells at $32{ }^{\circ} \mathrm{C}$ also resulted in the expected subsequent 184 shift of VSVG-GFP from the Golgi to the PM.

185 We predicted that if NleF disrupts Tmp21 function, VSVG-GFP redistribution to the Golgi would 186 either be delayed or blocked by NleF. After transfecting NleF and performing temperature shift 187 experiments, we observed that VSVG-GFP was retained in the ER at $19{ }^{\circ} \mathrm{C}$ in $74 \%$ of cells examined, 188 rather than redistributing to the Golgi ( $p<0.05$, Fisher's exact test; Fig. 3D). Similarly, in the presence of 189 NleF, VSVG-GFP was mislocalized to the Golgi at $32{ }^{\circ} \mathrm{C}$ in $58 \%$ of cells examined, rather than trafficking 190 to the PM ( $p<0.05$, Fisher's exact test; Fig. 3E).

191

192 Conclusions. Overall, the yeast two-hybrid, immunoprecipitation, and BiFC data suggest that NleF binds 193 to Tmp21. Expressing NleF disrupted VSVG-GFP localization, suggesting that the NleF-Tmp21 interaction 194 disrupts intracellular protein trafficking. The functional significance of these data in subverting host cells in 195 the context of $E$. coli and C. rodentium infection awaits further experimentation.

197 Acknowledgments. The project described was supported by Grant Number Al087923 from the National 198 Institute of Allergy and Infectious Diseases (NIAID). Its contents are solely the responsibility of the authors 199 and do not necessarily represent the official views of the NIAID. 
References.

202 Aguilera-Romero, A., Kaminska, J., Spang, A., Riezman, H., Muniz, M., 2008. The yeast p24 complex is required for the formation of COPI retrograde transport vesicles from the Golgi apparatus. The

Anantharaman, V., Aravind, L., 2002. The GOLD domain, a novel protein module involved in Golgi function and secretion. Genome biology 3, research0023.

Blum, R., Lepier, A., 2008. The luminal domain of p23 (Tmp21) plays a critical role in p23 cell surface trafficking. Traffic (Copenhagen, Denmark) 9, 1530-1550. localization and in vivo trafficking of p24A and p23. Journal of cell science 112 ( Pt 4), 537-548. 1673-1678.

Blum, R., Pfeiffer, F., Feick, P., Nastainczyk, W., Kohler, B., Schafer, K.H., Schulz, I., 1999. Intracellular

Celli, J., Salcedo, S.P., Gorvel, J.P., 2005. Brucella coopts the small GTPase Sar1 for intracellular replication. Proceedings of the National Academy of Sciences of the United States of America 102,

Clarke, S.C., 2001. Diarrhoeagenic Escherichia coli--an emerging problem? Diagnostic microbiology and infectious disease 41, 93-98.

Coombes, B.K., Wickham, M.E., Mascarenhas, M., Gruenheid, S., Finlay, B.B., Karmali, M.A., 2008. Molecular analysis as an aid to assess the public health risk of non-O157 Shiga toxin-producing Escherichia coli strains. Applied and environmental microbiology 74, 2153-2160.

Cornelis, G.R., 2010. The type III secretion injectisome, a complex nanomachine for intracellular 'toxin' delivery. Biological chemistry 391, 745-751.

Deng, W., Puente, J.L., Gruenheid, S., Li, Y., Vallance, B.A., Vazquez, A., Barba, J., Ibarra, J.A., O'Donnell, P., Metalnikov, P., Ashman, K., Lee, S., Goode, D., Pawson, T., Finlay, B.B., 2004. Dissecting virulence: systematic and functional analyses of a pathogenicity island. Proceedings of the National Academy of Sciences of the United States of America 101, 3597-3602.

Echtenkamp, F., Deng, W., Wickham, M.E., Vazquez, A., Puente, J.L., Thanabalasuriar, A., Gruenheid, S., Finlay, B.B., Hardwidge, P.R., 2008. Characterization of the NleF effector protein from attaching and effacing bacterial pathogens. FEMS microbiology letters 281, 98-107. 
Gao, X., Wan, F., Mateo, K., Callegari, E., Wang, D., Deng, W., Puente, J., Li, F., Chaussee, M.S., Finlay, B.B., Lenardo, M.J., Hardwidge, P.R., 2009. Bacterial effector binding to ribosomal protein s3

Hu, C.D., Chinenov, Y., Kerppola, T.K., 2002. Visualization of interactions among bZIP and Rel family proteins in living cells using bimolecular fluorescence complementation. Molecular cell 9, 789-798.

Kaiser, C., Ferro-Novick, S., 1998. Transport from the endoplasmic reticulum to the Golgi. Current opinion in cell biology 10, 477-482.

Kim, J., Thanabalasuriar, A., Chaworth-Musters, T., Fromme, J.C., Frey, E.A., Lario, P.I., Metalnikov, P., Rizg, K., Thomas, N.A., Lee, S.F., Hartland, E.L., Hardwidge, P.R., Pawson, T., Strynadka, N.C., Finlay, B.B., Schekman, R., Gruenheid, S., 2007. The bacterial virulence factor NleA inhibits cellular protein secretion by disrupting mammalian COPII function. Cell Host Microbe 2, 160-171.

Kuhle, V., Abrahams, G.L., Hensel, M., 2006. Intracellular Salmonella enterica redirect exocytic transport processes in a Salmonella pathogenicity island 2-dependent manner. Traffic (Copenhagen, Denmark) 7, 716-730.

Lippincott-Schwartz, J., Roberts, T.H., Hirschberg, K., 2000. Secretory protein trafficking and organelle dynamics in living cells. Annual review of cell and developmental biology 16, 557-589.

Orci, L., Ravazzola, M., Volchuk, A., Engel, T., Gmachl, M., Amherdt, M., Perrelet, A., Sollner, T.H., Rothman, J.E., 2000. Anterograde flow of cargo across the golgi stack potentially mediated via bidirectional "percolating" COPI vesicles. Proceedings of the National Academy of Sciences of the United States of America 97, 10400-10405.

Presley, J.F., Cole, N.B., Schroer, T.A., Hirschberg, K., Zaal, K.J., Lippincott-Schwartz, J., 1997. ER-toGolgi transport visualized in living cells. Nature 389, 81-85.

Toomre, D., Keller, P., White, J., Olivo, J.C., Simons, K., 1999. Dual-color visualization of trans-Golgi network to plasma membrane traffic along microtubules in living cells. Journal of cell science 112 ( Pt 1), 21-33. 
256 Wessels, E., Duijsings, D., Niu, T.K., Neumann, S., Oorschot, V.M., de Lange, F., Lanke, K.H., 257 Klumperman, J., Henke, A., Jackson, C.L., Melchers, W.J., van Kuppeveld, F.J., 2006. A viral 258 protein that blocks Arf1-mediated COP-I assembly by inhibiting the guanine nucleotide exchange 259 factor GBF1. Developmental cell 11, 191-201.

260 Wessels, E., Duijsings, D., Notebaart, R.A., Melchers, W.J., van Kuppeveld, F.J., 2005. A proline-rich 261 region in the coxsackievirus 3A protein is required for the protein to inhibit endoplasmic reticulum262 to-golgi transport. Journal of virology $79,5163-5173$.

263 Zhang, Y., Laing, C., Steele, M., Ziebell, K., Johnson, R., Benson, A.K., Taboada, E., Gannon, V.P., 2007. 264 Genome evolution in major Escherichia coli O157:H7 lineages. BMC genomics 8, 121.

265

266

267 

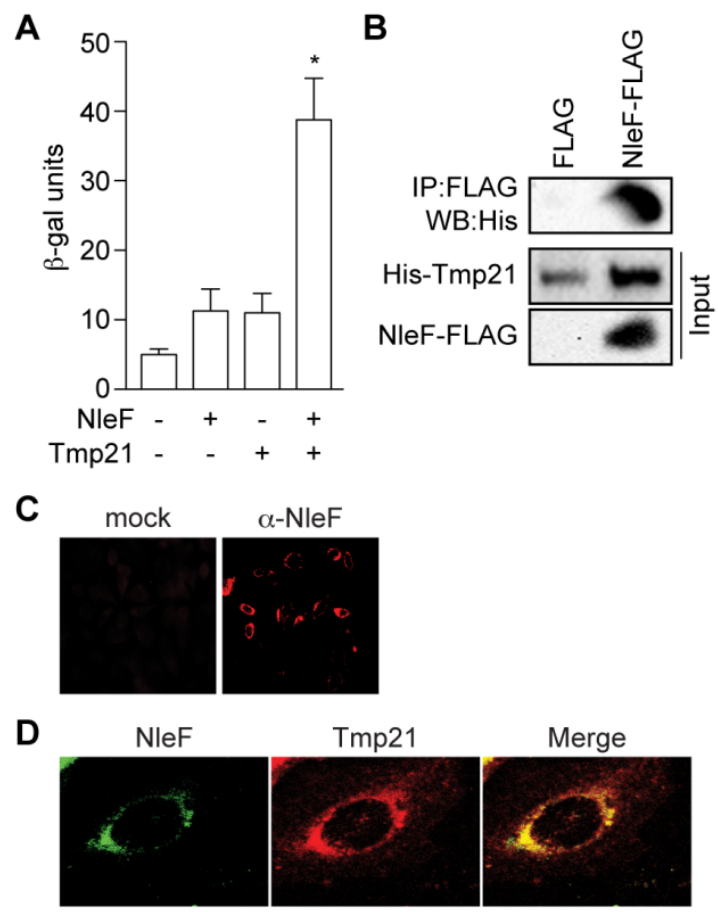

270 Figure 1. NleF binds Tmp21. A. $\beta$-galactosidase assays. NleF was expressed in yeast in the presence

271 or absence of human Tmp21 and $\beta$-galactosidase activity was quantified. Asterisks indicate significantly

272 different $\beta$-galactosidase activity as compared with untransformed yeast $(p<0.05$, t-test). B. Co-

273 immunoprecipitation. His-Tmp21 and was co-expressed with NleF-FLAG in E. coli BL21(DE3). NleF was

274 immunoprecipitated and its binding to His-Tmp21 binding was assessed using immunoblotting. C.

275 Immunofluorescence microscopy. HeLa cells were infected with C. rodentium DBS100/pnleF-FLAG

276 and stained with an $\alpha-N l e F$ antibody (red). D. NleF and Tmp21 co-localize. HeLa cells were infected with

277 C. rodentium/pnleF-FLAG, transfected with Tmp21-HA and stained with $\alpha-\mathrm{NleF}$ (green) and $\alpha-\mathrm{HA}$ (red)

278 antibodies.

279 

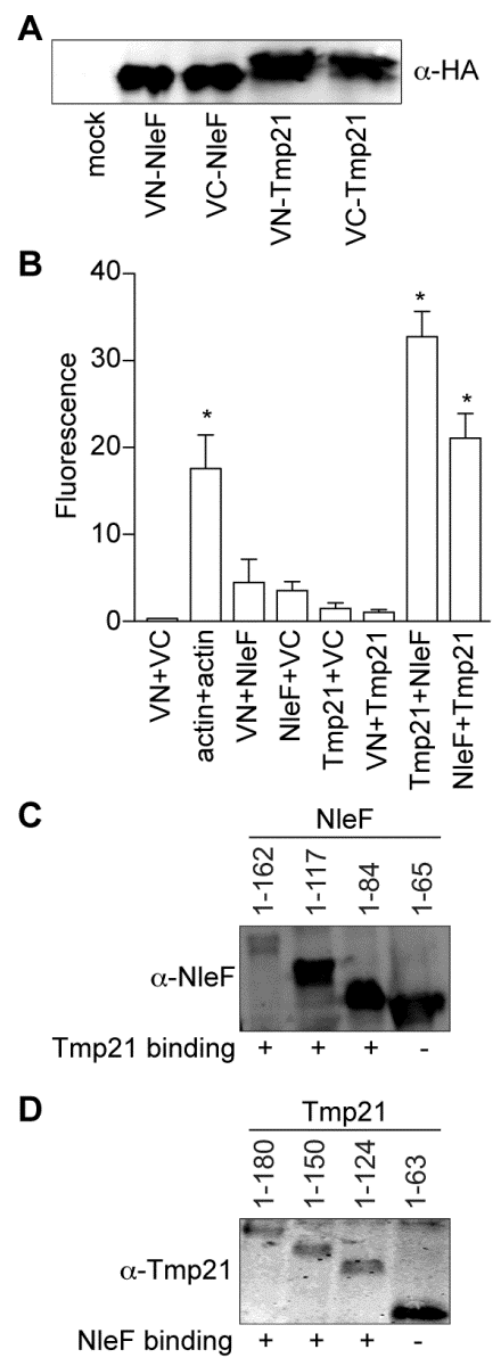

281 Figure 2. BiFC. A. NleF and Tmp-21 expression. NleF and Tmp21 were cloned into eYFP-VN and 282 eYFP-VC vectors and protein expression was evaluated by immunoblotting. B. BiFC quantification. 283 Relative fluorescence intensity after co-transfecting indicated NleF- and Tmp21-eYFP plasmid 284 combinations $(n=3)$. Asterisks indicate significantly different fluorescence intensity as compared with 285 untransfected samples ( $p<0.05$, ANOVA). C. NleF truncations binding to Tmp21. NleF truncations 286 were cloned into eYFP-VC vectors and protein expression was evaluated by immunoblotting. Binding to 287 Tmp21-eYFP-VN was measured using BiFC and is scored as positive (+) or negative (-) in the respective 288 columns. D. Tmp21 truncations binding to NleF. Tmp21 truncations were cloned into eYFP-VC vectors 289 and protein expression was evaluated by immunoblotting. Binding to NleF-eYFP-VN was measured using 290 BiFC and is scored as positive (+) or negative in the respective columns. 
A

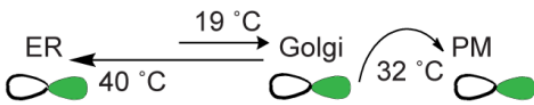

B

Calnexin VSVG Merge

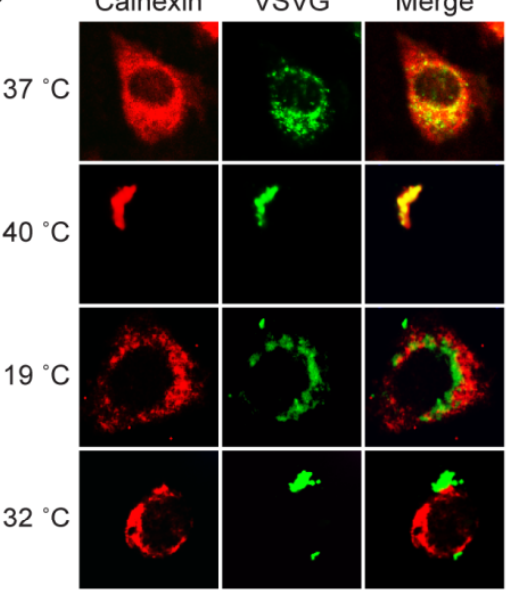

D

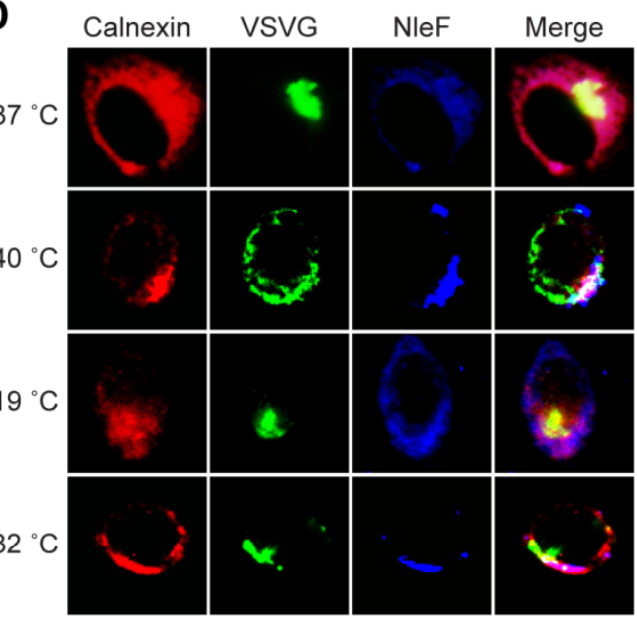

C

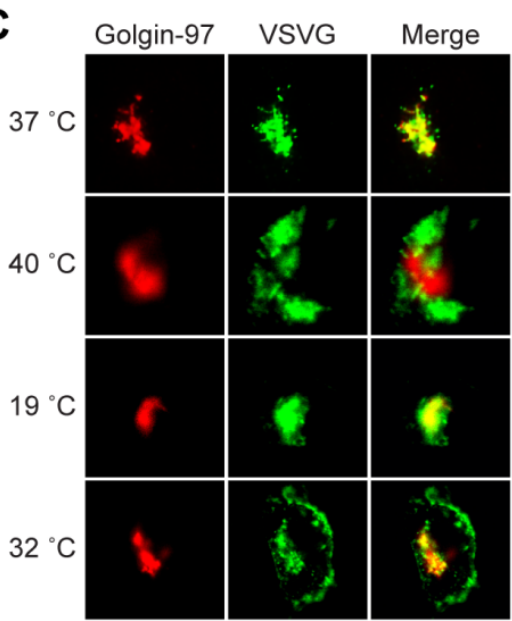

E

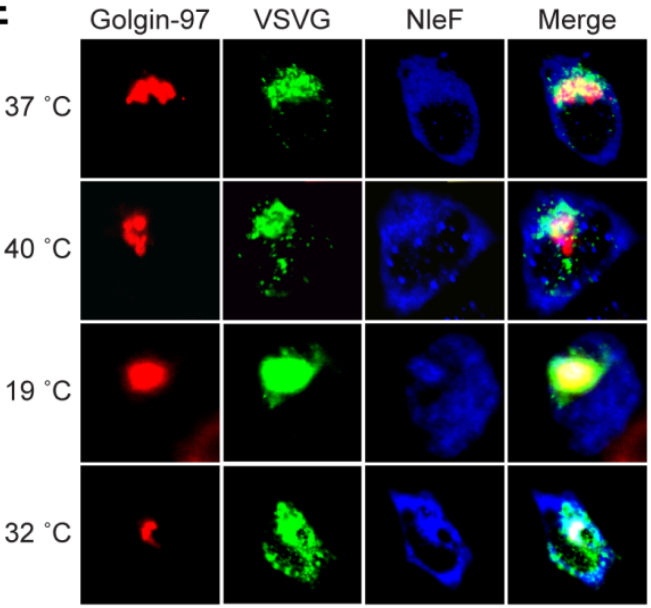

294 Figure 3. NleF alters VSVG-GFP trafficking. A. VSVG-GFP localization schematic. At $40{ }^{\circ} \mathrm{C}$, VSVG-

295 GFP becomes reversibly misfolded and retained in the ER. Incubation at $19{ }^{\circ} \mathrm{C}$ allows VSVG-GFP

296 refolding and transport to the TGN. Further incubation at $32{ }^{\circ} \mathrm{C}$ allows VSVG-GFP trafficking to the PM. B.

297 VSVG-GFP colocalization with calnexin. HeLa cells were transfected with VSVG-GFP and then

298 incubated at $37{ }^{\circ} \mathrm{C}$ or shifted to $19{ }^{\circ} \mathrm{C}, 32{ }^{\circ} \mathrm{C}$, or $40{ }^{\circ} \mathrm{C}$. Cells were stained with an $\alpha$-calnexin antibody. $\mathrm{C}$.

299 VSVG-GFP colocalization with golgin-97. Experiment performed as in (B). Cells were stained with an $\alpha-$

300 golgin-97 antibody. D. Impact of NleF on VSVG-GFP colocalization with calnexin. HeLa cells were

301 cotransfected with both VSVG-GFP and NleF-HA and then incubated at $37{ }^{\circ} \mathrm{C}$ or shifted to $19^{\circ} \mathrm{C}, 32{ }^{\circ} \mathrm{C}$, or

$30240{ }^{\circ} \mathrm{C}$. Cells were stained with $\alpha$-calnexin (red) and $\alpha$-HA (blue) antibodies. E. Impact of NleF on VSVG-

303 GFP colocalization with golgin-97. Experiment performed as in (D). Cells were stained with $\alpha-g o l g i n-97$ 304 (red) and $\alpha-\mathrm{HA}$ (blue) antibodies. 


\begin{tabular}{|c|c|c|}
\hline Strain or plasmid & Description & Reference \\
\hline \multicolumn{3}{|l|}{ Yeast strains } \\
\hline S. cerevisiae $\mathrm{AH} 109$ & $\begin{array}{l}\text { MATa,trp1-901,leu2-3,112,ura3-52, his3- } \\
\text { 200,gal4D, gal80D,LYS2::GAL1 }{ }_{\text {UAS }}-G A L 1_{\text {TATA }}-H I S 3, G A L 2_{U A S}-G A L 2_{T_{A T A}^{-}} \\
\text {ADE2,URA3::MEL1 } 1_{\text {UAS }}-M E L 1_{\text {TATA }}-\text { lacZ }\end{array}$ & Clontech \\
\hline S. cerevisiae Y187 & $\begin{array}{l}\text { MAT } \alpha \text {,ura3-52, his3-200, ade2-101,trp1-901,leu2-3,112,gal4D, met } \\
\text {,gal80D,URA3::GAL1 }{ }_{\text {UAS }}-G A L 1_{\text {TATA }}-\text { lacZ }\end{array}$ & Clontech \\
\hline yPRH-5 & HeLa cDNA library in Y187 & Clontech \\
\hline yPRH-11 & AH109/nleF-Gal4 ${ }_{D B D}-M y c$ & This study \\
\hline \multicolumn{3}{|l|}{ Bacterial strains } \\
\hline C. rodentium DBS100/NleF-FLAG & C. rodentium ATCC 51459 expressing FLAG-tagged NleF & This study \\
\hline E. coli BL21(DE3) & E. coli $\mathrm{F}^{-}$ompt hsdSB $\left(\mathrm{r}_{\mathrm{B}}^{-} \mathrm{m}_{\mathrm{B}}^{-}\right) \mathrm{gal} d \mathrm{dcm}(\mathrm{DE} 3)$ & Novagen \\
\hline E. coli BL21(DE3)/NleF-FLAG & NleF-FLAG & This study \\
\hline E. coli BL21(DE3)/Tmp21-His & Tmp21-His & This study \\
\hline E. coli BL21(DE3)/NleF-FLAG/Tmp21-His & NleF-FLAG + Tmp21-His coexpression & This study \\
\hline \multicolumn{3}{|l|}{ Plasmids } \\
\hline pGBKT7 & Two-hybrid bait plasmid & Clontech \\
\hline pGADT7 & Two-hybrid library plasmid & Clontech \\
\hline nleF-Gal4 DBD-Myc & NleF-Gal4-Myc bait plasmid & This study \\
\hline pFLAG-CTC & Bacterial FLAG fusion protein expression & Sigma \\
\hline NleF-pFLAG-CTC & NleF-FLAG & (Echtenkamp et al., 2008) \\
\hline pET28a & Bacterial hexahistidine fusion protein expression & Novagen \\
\hline Tmp21-pET28a & Tmp21-His & This study \\
\hline VN & Venus fluorescence protein (AAs 1-173) & (Gao et al., 2009) \\
\hline VC & Venus fluorescence protein (AAs 155-238) & (Gao et al., 2009) \\
\hline VN-actin & Venus 1-173 fused to human actin & (Gao et al., 2009) \\
\hline VC-actin & Venus $155-238$ fused to human actin & (Gao et al., 2009) \\
\hline VN-NleF & Venus $1-173$ fused to NleF & This study \\
\hline VC-NleF & Venus $155-238$ fused to NleF & This study \\
\hline VC-NleF (1-162) & Venus $155-238$ fused to NleF (AAs 1-162) & This study \\
\hline VC-NleF (1-117) & Venus $155-238$ fused to NleF (AAs 1-117) & This study \\
\hline VC-NleF (1-84) & Venus $155-238$ fused to NleF (AAs 1-84) & This study \\
\hline VC-NleF (1-65) & Venus $155-238$ fused to NleF (AAs 1-65) & This study \\
\hline VN-Tmp21 & Venus $1-173$ fused to Tmp21 & This study \\
\hline VC-Tmp21 & Venus $155-238$ fused to Tmp21 & This study \\
\hline VC-Tmp21 (1-180) & Venus 155-238 fused to Tmp21 (AAs 1-180) & This study \\
\hline VC-Tmp21 (1-150) & Venus $155-238$ fused to Tmp21 (AAs 1-150) & This study \\
\hline VC-Tmp21 (1-124) & Venus 155-238 fused to Tmp21 (AAs 1-124) & This study \\
\hline VC-Tmp21 (1-63) & Venus 155-238 fused to Tmp21 (AAs 1-63) & This study \\
\hline pEGFP-VSVG & VSVG in pEGFP-N1 & (Presley et al., 1997) \\
\hline
\end{tabular}

